\title{
Properties of a Self - gain Optical Fiber Differential Pressure Sensor Based on Damping Piston
}

\author{
Hao Hu and Liqiong Zhong* \\ College of Mechanical engineering, Guiyang college, Guiyang 550003,China \\ College of Mechanical engineering, Guizhou university, Guiyang 550025,China \\ ${ }^{*}$ Corresponding author
}

\begin{abstract}
In this paper, the performance of a self-gain optical fiber differential pressure sensor based on damping piston is studied. Firstly, proposes the structure of mechanical packaging sensor probe based on the damping piston, and designs the sensor system; then, it carries out the theoretical research on the sensor and builds the intensity compensation modulation of the sensor. Finally, the experimental model machine is used for the strength compensation test and the differential pressure test. The experiment results show that after the intensity compensation of sensor, the output value can be maintained at a constant value, which is not affected by the fluctuation of light source power, and the output value can achieve the gain; the sensor of damping spring in different designed sizes has different detection range. When the detection range is $[-80 \mathrm{KPa}, 80 \mathrm{KPa}]$, the sensitivity is about $0.024 / \mathrm{KPa}$ and the linearity is about $6 \%$, with a good sensitivity and linearity. The research shows that the optical fiber differential pressure sensor has better detection performance and can meet the needs of different detection occasions.
\end{abstract}

Keywords-properties; damping piston; self-gain; geometric optics; optical fiber; differential pressure sensor

\section{INTRODUCTION}

With the advance and development of modern industrial technology, the differential pressure sensor has been applied in many high-precision detection occasions. From the micro-flow measurement, leakage test, clean room monitoring and environmental sealing test to the measurement of pressure field distribution, air quantity measurement, measurement of artificial respirator's respiratory capacity and measurement of micro-pressure difference between the rooms, all need a stable and reliable differential pressure sensor. Besides, with the development of modern detection technology, the application of differential pressure sensor will be further expanded, and the requirements for the differential pressure sensor technology will be increasingly high. Because of this, many scholars are committed to the improvement of differential pressure sensor's structure and advance of its performance in order to make it better serve the field of modern industrial detection [1-5].

Nowadays, the differential pressure sensor is mainly two kinds, respectively pressure resistance type and capacitance type. There are more prominent contradictions between sensitivity and frequency response of pressure resistance type, and the temperature has larger effect on the performance of this kind of sensor; for capacitance type, the parasitic capacitance and distributed capacitance have effect on sensitivity and measuring accuracy, and there is complex circuit connected with the sensor and other deficiencies. It is because of so many shortcomings of traditional pressure sensor, the scholars both at home and abroad started to study some new differential pressure sensors, for example Dong Guoqiang et al conducts research on the micro-differential pressure sensor with the use of magnetic liquid [6], depending on the displacement process of magnetic fluid under pressure to generate an electrical signal to realize the detection of the pressure, and it has limitations in application; Hao-Jan Sheng of Taiwan and Jose.L.A.V of America et al each put forward a kind of optical fiber differential pressure sensor structure based on bragg grating [78]. The differential pressure sensor with such optical fiber grating has high degree of sensitivity but the structure is complex and the cost is very high, so it is not conducive to the promotion. But the intensity modulation as a widely used modulation method in optical fiber sensor technology has been studied by some scholars [9-10], but the research literature on optical fiber differential pressure sensor based on intensity modulation is very few. The Japanese Seiichiro Kinugasa proposes a conception of reflective optical fiber differential pressure sensor using the change of the reflector and the distance of the optical fiber to detect external differential pressure; Tong Chengguo designs the optical fiber differential pressure sensor with a double C-type spring tube, and based on the principle of intensity modulation, the differential pressure is detected in two double C-type spring tubes.

Under this background, this paper proposes a self gainboosted differential pressure sensor based on the damping piston, and studies its structure and performance, in order to make the research on the differential pressure sensor further expanded and push the development of the design and promotion of new differential pressure sensor.

\section{SENSOR STRUCTURE}

As shown in Figure 1, the sensor comprises a probe part and the structure of self-compensating double light path of single light source. The light emitted by the light source $S$ through the $\mathrm{Y}$ coupler is divided into two paths. The one enters the probe $M_{1}$, and after reflection reaches the photoelectric detector $D_{1}$; the other enters the probe $M_{2}$, and after reflection reaches the photoelectric detector $D_{2}$. 


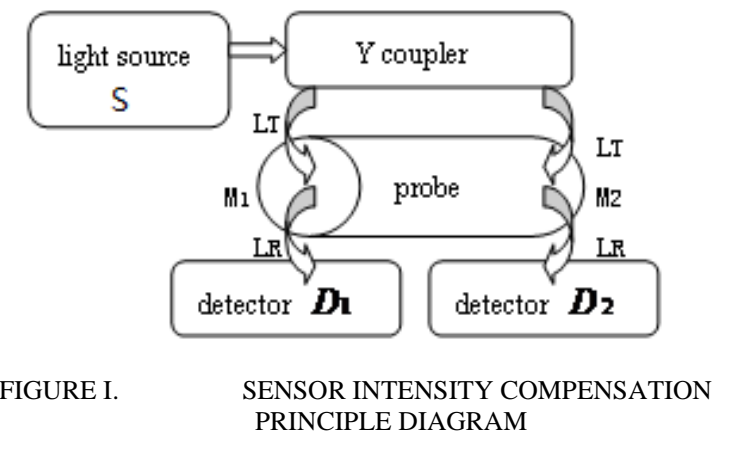

As shown in Figure 2 below, the structure of sensor probe is made up of the end cover, cylinder, piston, spring, high light transmission layer, reflective layer and other parts. When the sensor is working, if there is differential pressure for the fluid on the detection cavities of both sides of the piston, the piston is out of balance and slides towards one side. The damping spring is compressed in this side, and the damping spring of the other side is stretched. With the damping spring compressed, the distance between optical fiber bundle and the reflector of piston surface decreases, and the signal intensity of the output light receiving optical fiber is weakened. With the damping spring stretched, the distance between optical fiber bundle and the reflector of piston surface increases, and the signal intensity of the output light receiving optical fiber is enhanced. After the photoelectric conversion and signal processing, the output value will increase exponentially, thus detection sensitivity is improved and the self-gain effect is achieved. Through the change of the output value, it is realized that the fluid differential pressure between two detection cavities can be judged, and the detection of differential pressure in different positions is realized; if the fluid pressure in the two detection cavities is equal and the piston does not produce axial displacement, then the signal intensity of output light of the fiber received by two fiber bundles is equal, and after the calculation of the photoelectric conversion and signal processing, the output value is 1 .

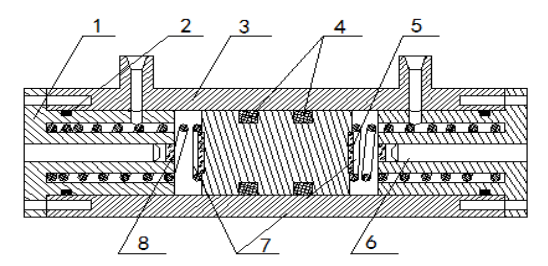

1 end cap 2 sealing ring 3 cylinder 4 sealing ring 5 reflector 6 optical fiber probe 7 damping spring 8 high transmission film

FIGURE II.

PROBE STRUCTURE

\section{TEST RESUlts AND ANALYSIS}

The damping piston optical fiber differential pressure sensor is used for the intensity compensation test. In the test, the light intensity detection system is used to detect the output electrical signal of the probe of two sensors. The light source adopts a semiconductor laser LD, with 1310nm of output peak wavelength. The photoelectric detector uses PIN photodiode, with the working wavelength range from $1100 \mathrm{~nm}$ to $1650 \mathrm{~nm}$, the maximum dark current as $1 \mathrm{nA}$, the minimum optical response degree as $0.85 \mathrm{~A} / \mathrm{W}$, and the response time as $0.1 \mathrm{~ns}$. Through the alternate control of circuits, the output power of light source is changed constantly, the output power of light source are respectively $100 \%, 70 \%$ and $40 \%$. When the differential pressure between the two sides of sensor are $0 \mathrm{KPa}$, $20 \mathrm{KPa}$ and $40 \mathrm{KPa}$, the size of output signal of photoelectric detector D1 and D2 is recorded. The experimental results are shown in Figure 3, Figure 4 and Figure 5:

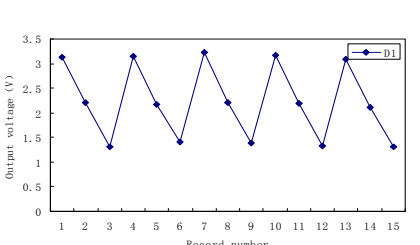

(a) D1 output voltage

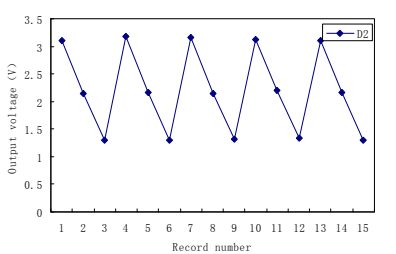

(b) D2 output voltage

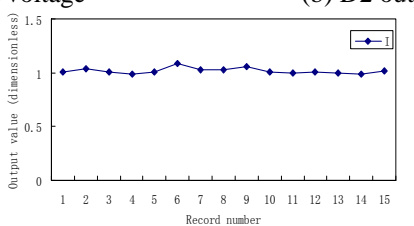

(c) Output value

FIGURE III. IN THE DIFFERENTIAL PRESSURE OKPA, D1 AND D2 OUTPUT VOLTAGE AND COMPENSATION OUTPUT VALUE I

From the above Figure 3 (a) and (b), it is not difficult to see that when the differential pressure of sensor is detect as $0 \mathrm{KPa}$, the output power of light source changes and output voltage value of photoelectric detector D1 and D2 will have greater fluctuation. When the output power of light source is $100 \%$, the output voltage is about $3.1-3.2 \mathrm{~V}$. When the output power of light source is $70 \%$, the output voltage is about $2.1-2.2 \mathrm{~V}$. When the output power of light source is $40 \%$, the output voltage is about $1.3-1.4 \mathrm{~V}$. If the intensity compensation is not carried out on it, the final detection value will not be correct. From the above Figure 3 (c), is not difficult to see that after the intensity compensation of sensor in the process of the change of the light source output power, the output value is maintained at constant value near 1 , which will not fluctuate with the power fluctuation of the light source, that is when the differential pressure on both sides of sensor is $0 \mathrm{KPa}$, the detection value of sensor is 1 .

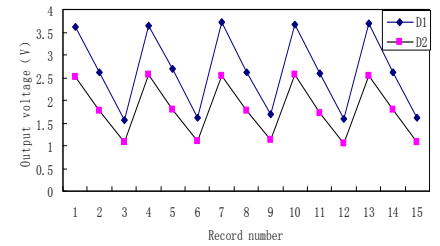

(a) D1, D2 output voltage

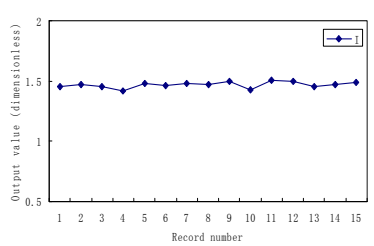

(b) Output value

FIGURE IV. IN THE DIFFERENTIAL PRESSURE 20KPA, D1 AND D2 OUTPUT VOLTAGE AND COMPENSATION OUTPUT VALUE I 


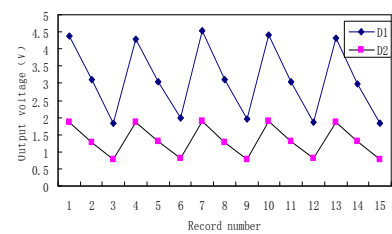

(a) D1, D2 output voltage

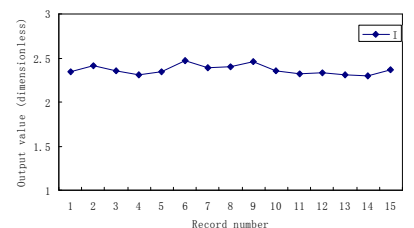

(b) Output value
FIGURE V. IN THE DIFFERENTIAL PRESSURE 40KPA, D1 AND D2 OUTPUT VOLTAGE AND COMPENSATION OUTPUT

VALUE I

From Figure 4(a) and Figure 5 (a), it is not difficult to see that when the differential pressure of sensor are detected are respectively $20 \mathrm{KPa}, 40 \mathrm{KPa}$, the output power of light source changes and the output voltage value of photoelectric detector D1 and D2 also has large fluctuations. In the Figure 4 (a) and Figure 5 (a), the above toothed curve is the output voltage of photoelectric detector $\mathrm{D} 1$, and the below toothed curve is the output voltage of photoelectric detector D2. It is not difficult to see that in light of the same output power of light source, the output voltage of D1 increases with the increase of detected differential pressure and the output voltage of D2 decreases with the decrease of detected differential pressure. This is because the initial working state of the sensor is in the middle position of the curve of the intensity modulation curve. When the differential pressure effect is received, the output voltage of the low voltage side decreases and the output voltage of the high voltage side increases. From above Figure 4(b) and Figure 5 (b), it is not difficult to see that after the intensity compensation of the sensor, when the output power of light source changes, the output value is maintained about from 1.5 to 2.4, which will not fluctuate with the power fluctuation of the light source, that is when the differential pressure of the sensor on both sides is respectively $20 \mathrm{KPa}$ and $40 \mathrm{KPa}$, the detection value of sensor is 1.5 and 2.4 , reaching the intensity compensation effect. The increase rate of the output value in the three kinds of differential pressure is $50 \%$ and $60 \%$ respectively, and it will increase greatly with the increase of differential pressure and thus the gain effect can be achieved.

According to the above design and theoretical analysis, the experimental prototype of optical fiber differential pressure sensor based on damping piston is developed. The prototype is composed of the following key components: stabilized voltage supply, a light source of semiconductor laser, the optical fiber probe (multimode optical fiber) integrated with a incident optical fiber and a receiving optical fiber, mechanical packaged sensor probe, damping spring with certain rigidity and the photodiode. The photodiode is connected with the signal detection module of light intensity, and then connected with the A/D conversion module. After the pre-processing of photoelectric signal, then it is input to the computer for the calculation of the data at the later stage. Through the detection experiment of differential pressure, the results are obtained as following Figure 6.

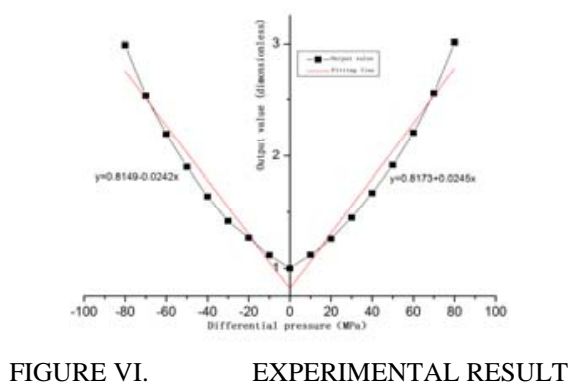

The Figure 6 above shows the output value changes of the sensor when the differential pressure between the two detection cavities of sensor are respectively $-80 \mathrm{KPa},-70 \mathrm{KPa},-60 \mathrm{KPa}$, $50 \mathrm{KPa},-40 \mathrm{KPa},-30 \mathrm{KPa},-20 \mathrm{KPa},-10 \mathrm{KPa}, 0 \mathrm{KPa}, 10 \mathrm{KPa}$, 20KPa, 30KPa, $40 \mathrm{KPa}, 50 \mathrm{KPa}, 60 \mathrm{KPa}, 70 \mathrm{KPa}$ and $80 \mathrm{KPa}$. It is not difficult to see from the figure that in the damping spring with the current rigidity, the sensor's detection range is [-80KPa, $80 \mathrm{KPa}$ ] and the change of the rigidity of the damping spring is used to change the sensor's detection range.

Then, the sensitivity of the sensor is about $0.024 / \mathrm{KPa}$ and the linearity is about $6 \%$, which has high sensitivity and good linearity, and is consistent with the theoretical analysis.

Before you begin to format your paper, first write and save the content as a separate text file. Keep your text and graphic files separate until after the text has been formatted and styled. Do not use hard tabs, and limit use of hard returns to only one return at the end of a paragraph. Do not add any kind of pagination anywhere in the paper. Do not number text headsthe template will do that for you.

Finally, complete content and organizational editing before formatting. Please take note of the following items when proofreading spelling and grammar:

\section{CONCLUSIONS}

This paper presents a new type of damping piston self-gain optical fiber differential pressure sensor. First, the probe structure of the differential pressure sensor and the light path structure of the sensor are designed. The probe of sensor is a mechanical packaged structure based on damping piston, and the light path of the sensor is the self-compensating double light path structure of single source, so as to realize the new type of optical fiber differential pressure sensor; then the paper analyzes the intensity compensation model of the sensor theoretically. the output signal after the intensity compensation of the sensor can effectively eliminate the power fluctuation of light source and the error of coupler, and then the ratio operation is conducted on the output value of two detection cavities of the sensor, which can better realize the self gain and self compensation of the sensor; Finally, the damping piston optical fiber differential pressure sensor is used for the intensity compensation test and differential pressure test. Through the intensity compensation test, it can be known that when the changes occur in the power of light source, the output voltage of photoelectric detector will have larger fluctuation, and that after intensity compensation, the output value can be maintained at a constant value, which will not fluctuate with the power fluctuation of the light source, that is the sensor 
achieves better intensity compensation effect. Through the detection experiment of differential pressure, it can be known that for the damping spring with certain rigidity and the sensor with certain detection range, the detection range of the sensor can be changed by changing the rigidity of damping spring, and that the sensor has high sensitivity and good linearity, consistent with experimental results and theoretical analysis.

\section{ACKNOWLEDGMENT}

Science and technology cooperation plan of Guizhou Province (LH[2015]7660); The introduction of talent fund of Guizhou University ((2014)43).

\section{REFERENCES}

[1] M. Shanmugavalli, G. Uma, M. Umapathy. Uncertainty Analysis Of Micro Differential Pressure Sensor Using Interval Analysis, J. International Journal On Smart Sensing And Intelligent Systems.2(2009)463-476.

[2] J. Albert Chiou, Steven Chen. Thermal hysteresis and voltage shift analysis for differential pressure sensors, J. Sensors and Actuators. 135(2007)107-112.

[3] Meenatchisundaram .S, Simha. Ashwin, Menon. Mukund Kumar, et al. Lumped parameter modeling of absolute and differential micro pressure sensors, J. Sensors and Transducers. 144(2012)76-91.

[4] Takahashi Hidetoshi, Dung Nguyen Minh, Matsumoto Kiyoshi1, et al. Differential pressure sensor using a piezoresistive cantilever, J. Journal of Micromechanics and Microengineering. 22(2012).

[5] Pavlik Michal, Haze Jiri, Vrba Radimir. Smart differential pressure sensor. International Conference on Informatics in Control, Automation and Robotics, Volume SPSMC,2007, pp: 244-248.

[6] DONG Guo qiang, LI De cai, HAO Rui can. Study on Micro Differential Pressure Sensor Based on Ferrofluid, J. CHINESE JOURNAL OF SENSORS AND ACTUATORS. 22(2009)50-53.

[7] Hao-Jan Sheng, Wen-Fung Liu,Kuei-Ru Lin,et al. High-sensitivity temperature-independent differential pressure sensor using fiber Bragg gratings, J. OPTICS EXPRESS. 16(2008)16014-16018.

[8] Jose L.A.V, Riode J.R.J, Manoel F da S, et al. U.S. Patent 2006/0062510 A1, (2006).

[9] Lars Rindorf, Poul Erik Hoiby, Jesper Bo Jensen,et al. Towards biochips using microstructured optical fiber sensors, J. Anal Bioanal Chem. 385(2006)1370-1375.

[10] TANG Yu, LIU Chuanju. Study on Optical Fiber Sensor and its Application, J. Science \& Technology Information. 7(2009)17-18. 